\title{
Corrosion Inhibition of N80 Steel by Newly Synthesized Imidazoline Based Ionic Liquid in 15\% HCl Medium: Experimental and Theoretical Investigations
}

\author{
Lei Guo ${ }^{1,2}$, Mengyue Zhu ${ }^{3}$, Jun Chang ${ }^{2, *}$, Renjith Thomas ${ }^{4}$, Renhui Zhang ${ }^{3}$, Pengjie Wang ${ }^{3}$, \\ Xingwen Zheng ${ }^{5}$, Yuanhua Lin ${ }^{1, *}$ Riadh Marzouki ${ }^{6,7}$ \\ ${ }^{1}$ State Key Laboratory of Oil and Gas Reservoir Geology and Exploitation, Southwest Petroleum \\ University, Chengdu 610500, Sichuan, China \\ ${ }^{2}$ School of Material and Chemical Engineering, Tongren University, Tongren 554300, China \\ ${ }^{3}$ School of Materials Science and Engineering, East China Jiaotong University, Nanchang 330013, \\ China \\ ${ }^{4}$ Department of Chemistry, St Berchmans College (Autonomous), Changanassery 686101, Kerala, \\ India \\ ${ }^{5}$ Key Laboratory of Material Corrosion and Protection of Sichuan Province, Zigong 643000, China \\ ${ }^{6}$ Chemistry Department, College of Science, King Khalid University, Abha 61413, Saudi Arabia \\ ${ }^{7}$ Chemistry Department, Faculty of Sciences, University of Sfax, 1171 Sfax 3000, Tunisia \\ *E-mail: junchang85@163.com, yhlin28@163.com
}

Received: 1 August 2021 / Accepted: 4 September 2021 / Published: 10 October 2021

\begin{abstract}
An imidazoline based ionic liquid, namely 2-heptadecyl-3-butyl-3-((n-methyl-thiourea) ethyl)-1Himidazole-3-ammonium (MTOI), was prepared by a simple synthesis route. The structure of MTOI was characterized by FTIR testing. Electrochemical techniques and surface analysis tests were used to explore the corrosion inhibition behavior of MTOI on N80 steel in $15 \% \mathrm{HCl}$ solution. The results showed that the adsorption of MTOI on the surface of N80 steel was in accordance with Langmuir isotherm, and it belongs to the mixed-type inhibitor with the maximum inhibition efficiency of $97 \%$ at $90 \mathrm{ppm}$. SEMEDS measurement verified that the $\mathrm{N}$ and $\mathrm{S}$ atoms in the ionic liquid were exactly adsorbed on the metal surface. Besides, the active nature of MTOI molecule was revealed by density functional theory (DFT) calculations, and the adsorption behavior of MTOI molecule on surface of N80 steel was elucidated by molecular dynamics (MD) simulations. This work can provide beneficial guidance for the future research of efficient and environmentally friendly ionic liquid corrosion inhibitors.
\end{abstract}

Keywords: Ionic liquid, Corrosion inhibition, N80 steel, Electrochemical techniques, Molecular simulation

\section{FULL TEXT}


(C) 2021 The Authors. Published by ESG (www.electrochemsci.org). This article is an open access article distributed under the terms and conditions of the Creative Commons Attribution license (http://creativecommons.org/licenses/by/4.0/). 